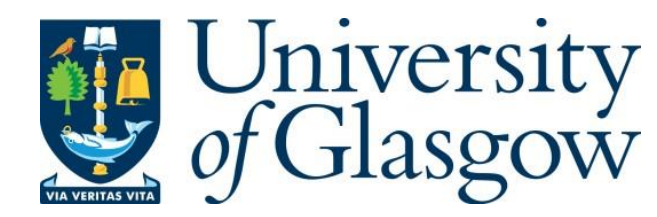

Pullanchiyodan, A., Manjakkal, L. and Dahiya, R. (2021) Metal coated fabric based asymmetric supercapacitor for wearable applications. IEEE Sensors Journal, (doi: 10.1109/JSEN.2021.3058894).

There may be differences between this version and the published version. You are advised to consult the publisher's version if you wish to cite from it.

http://eprints.gla.ac.uk/233679/

Deposited on: 9 February 2021

Enlighten - Research publications by members of the University of Glasgow http://eprints.gla.ac.uk 


\title{
Metal Coated Fabric Based Asymmetric Supercapacitor for Wearable Applications
}

\author{
Abhilash Pullanchiyodan, Libu Manjakkal, and Ravinder Dahiya, Fellow, IEEE
}

\begin{abstract}
This paper presents a fabric based asymmetric supercapacitor (ASC) with metal coated cloths as the electrode and the current collector. Graphite paste printed on silver coated textile (Berlin) is used as the negative electrode and nickel/copper/silver plated fabric (Nora Dell) as the positive electrode. Biocompatible polyvinyl alcohol (PVA)-potassium chloride $(\mathrm{KCl}) \mathrm{gel}$ is used as the electrolyte for ASC fabrication. During the electrochemical process, the metal oxides formed on the Nora Dell fabric contributes to the pseudocapacitance, whereas the graphite electrode forms the electric double layer capacitance. Compared to the Berlin/graphite based symmetric

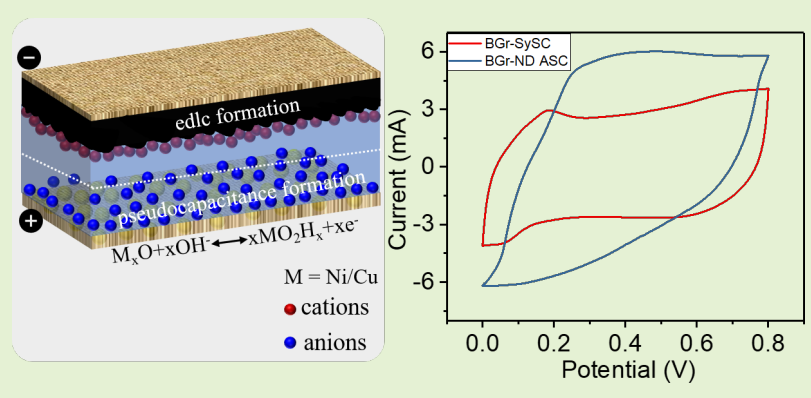

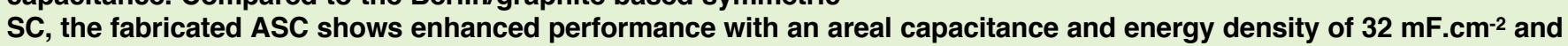
$2.8 \mu \mathrm{Wh} . \mathrm{cm}^{-2}$ respectively at a scan rate of $25 \mathrm{mV} . \mathrm{s}^{-1}$. The cyclic stability and self-charging capability of the device with flexible solar cells are also demonstrated as a proof of concept for use in practical applications. The presented energy storage device has potential to be used as a safe and sustainable alternative needed to meet the rapid growth in the field of wearable electronics.
\end{abstract}

Index Terms- Asymmetric supercapcitors, conductive fabrics, energy storage, metal oxides, wearables

\section{INTRODUCTION}

$\mathrm{R}_{\mathrm{s}}$ APID advances in areas such as wearable systems and eskin, with ever increasing number of devices, calls for safe and sustainable energy storage devices such as flexible supercapacitors (FSC) [1-5]. With high-power density $\left(\geq 10^{3}\right.$

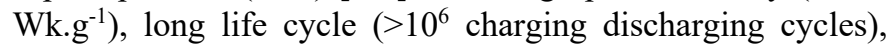
and low recharge time $(\sim 1$ minute) etc. the FSCs can power various devices on wearable systems [6-12]. Further, distinctive features such as flexibility, light weight, ecofriendly and biocompatibility make them attractive over the conventional Li-ion batteries. In context with wearable systems, the textile- based materials are most promising as they can be porous, environmentally friendly, flexible, and ease to integrate [13-17]. However, for a wearable textilebased SC fabrication, the investigation of electrochemical properties of the textile electrode with electrolytes is also important. Hence in textile based FSCs, the first task is to design the textile electrode including current collector and active materials.

An earlier version of this paper was presented at 2020 IEEE International Conference on Flexible and Printable Sensors and Systems (FLEPS) [DOI: 10.1109/FLEPS49123.2020.9239537]

This work was supported in part by the Engineering and Physical Sciences Research Council (EPSRC) Engineering Fellowship for Growth (EP/M002527/1 and EP/R029644/1), Royal Society-SERB Newton International Fellowship (NIF/R11182437), IEEE Electron Device Society through new initiative project on Circular Electronics, and European Commission through AQUASENSE project (H2020-MSCA-ITN-2018-813680).

The authors are with the Bendable Electronics and Sensing Technologies (BEST) Group, University of Glasgow, Glasgow G12 8QQ, U.K. (e-mail: ravinder.dahiya@glasgow.ac.uk).
The low energy density of FSCs is one area that requires greater attention and one of the solutions to this is to increase the specific capacitance [18]. In this regard the asymmetric SCs (ASC), which consists of two different types of electrode (negative and positive) can be helpful as they have presence of both the pseudocapacitance and the electric double layer capacitance (edlc) [3, 19-21]. The ASCs generally use carbon-based material such as graphene, carbon nanotube (CNT), graphite and their composites for negative electrode and the pseudocapacitive materials such as transition metal oxides and their composites as positive electrode [3, 21-25]. Some ASCs using on carbon and metal coated cloth have been reported too $[18,26]$. The high porosity of cloths is advantageous in terms of absorbance of aqueous active electrode ink and for converting the non-conductive cloth into conductive. The metal coated fabric shows better conductivity, scalability, flexibility, and excellent electrochemical performances compared with carbon cloths and as a result they are gaining attention for the fabrication of FSCs [1, 27].

Herein, we present a metal coated textile-based ASC for the first-time using graphite coated Berlin (silver coated silk) as the negative electrode and Nora Dell $(\mathrm{Ni} / \mathrm{Cu} / \mathrm{Ag}$ coated) fabric as the positive electrode. The Berlin fabric acts as the current collector and the graphite electrode is responsible for the edlc formation. On other hand, the Nora Dell fabric acts as the current collector as well as the positive electrode in such a way that the $\mathrm{Cu}$ and $\mathrm{Ni}$ undergo electrochemical oxidation process to from their respective metal oxide nanoparticles [1]. The binary metal oxides, formed in situ, acts as the pseudocapacitive material to improve the capacitance and energy density of the textile-based ASC [1]. The fabricated 
textile-based ASC shows a specific capacitance and energy density of $32 \mathrm{mF} . \mathrm{cm}^{-2}$ and $2.8 \mu \mathrm{Wh} . \mathrm{cm}^{-2}$ respectively at a scan rate of $25 \mathrm{mV} \cdot \mathrm{s}^{-1}$. This paper extends our IEEE FLEPS 2020 paper [28], where we presented graphite printed Berlin fabric based symmetric SC having an areal capacitance of 13.1 $\mathrm{mF} . \mathrm{cm}^{-2}$. The extended work presented in this paper include the fabrication of an asymmetric $\mathrm{SC}$ using the graphite printed Berlin fabric as the negative eelctrode and Nora dell fabric as the positve electrode.

This paper is organised as follows: Section II describes various materials and fabrication steps used for the development of ASC. The results are discussed in Section III and key outcomes are concluded in Section IV.

\section{EXPERIMENTAL}

\section{A. Materials}

Silver coated (Berlin) and $\mathrm{Ni} / \mathrm{Cu} / \mathrm{Ag}$ coated (Nora Dell) polyamide fabrics (Statex Produktions-und Vertriebs $\mathrm{GmbH}$ ) were used as the current collector/electrode material for FSC fabrication. Graphite (Sigma Aldrich), Triton X-100 (Sigma Aldrich), ethyl cellulose (Sigma Aldrich), terpineol (Sigma Aldrich) were used for the preparation of carbon electrode. A cellulose-polyester (Techni Cloth, TX 612) was also used as the separator. Potassium chloride $(\mathrm{KCl}$, Sigma Aldrich) and polyvinyl alcohol (PVA, Sigma Aldrich) were used for preparation of gel electrolyte.

\section{B. Fabrication of asymmetric supercapacitor}

Metal coated fabric-based ASC was fabricated using

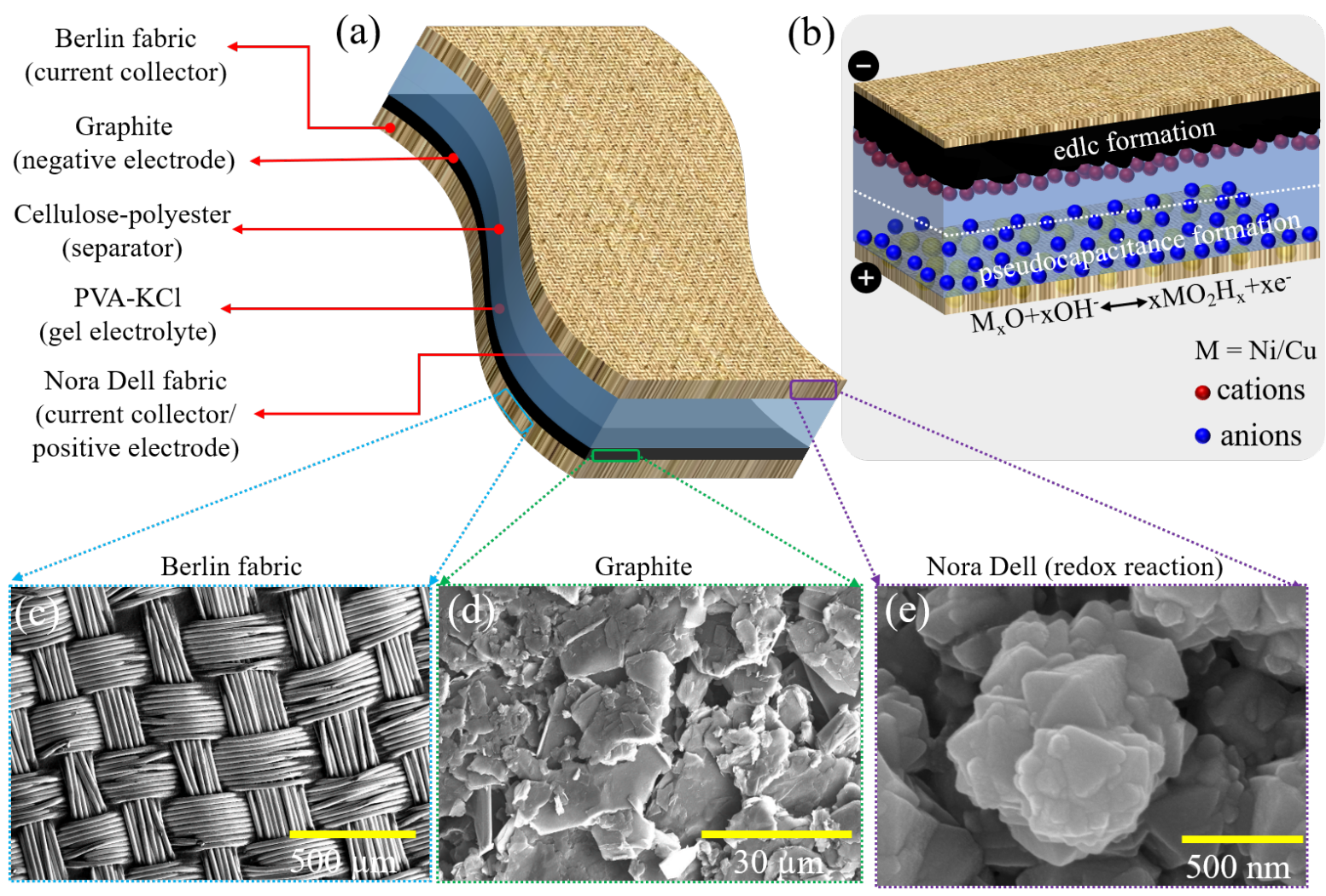

Fig. 1. (a) and (b) schematic representation of fabric ASC with different capacitance mechanism, microstructure of electrode/current collector materials (c) Berlin fabric, (d) graphite film and (e) Nora Dell fabric, respectively. graphite printed Berlin fabric as the negative electrode. To this end, a highly viscous graphite paste was formulated following previous reports $[1,28,29]$. In a typical procedure, $30 \mathrm{wt} . \%$ of graphite powder is mixed uniformly with Triton X-100 as the dispersant and ethyl cellulose as the binder in terpineol solvent. The printed electrode was dried in a hot air oven for about an hour at $80^{\circ} \mathrm{C}$. Similarly, the positive electrode was fabricated using Nora Dell textile. The biocompatible gel electrolyte for ASC was prepared using earlier report, which involves mixing $10 \mathrm{wt} . \%$ PVA solution with $\mathrm{KCl}$ solution at $80^{\circ} \mathrm{C}$ followed by vigorous stirring [1]. The prepared gel electrolyte was sandwiched between the positive and negative electrode and piece of cellulose-polyester cloth was used as the separator. A metal wire was used for external connection and it was fixed on the current collector using silver (Ag) conductive epoxy (from RS components, 186-3600) and insulated with polyurethane resin $[1,30]$. Fig. 1 shows the schematic representation of the developed ASC with individual components (Fig. 1(a)). Two different mechanism of charge storing such as edlc on negative electrode (graphite) and pseudocapacitance on positive (Nora Dell fabric) electrode was represented in Fig. 1(b).

\section{Characterization}

The fabrics (Berlin and Nora Dell) and printed graphite film were characterized by scanning electron microscopic analysis (SEM, FEI Nova NanoSem 630). The electrochemical studies such as cyclic voltammetry $(\mathrm{CV})$ in a potential range of 0 to $0.8 \mathrm{~V}$ with scan rate of $25-500 \mathrm{mV} \cdot \mathrm{s}^{-1}$ and electrochemical impedance spectroscopy (EIS) in the frequency range of 2 

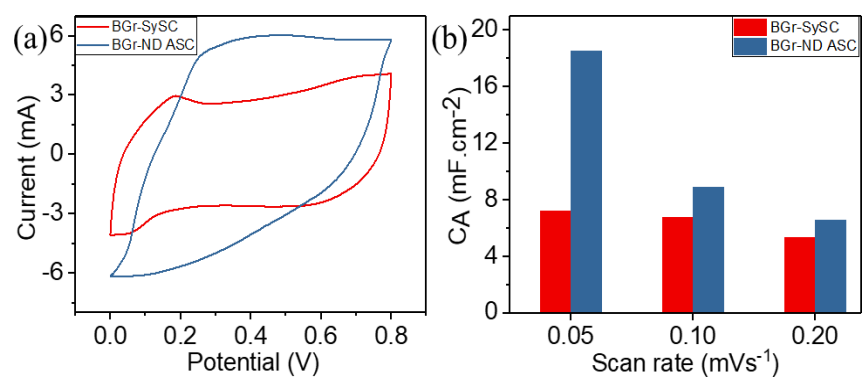

Fig. 2. Comparison of (a) CV curves at a scan rate of $100 \mathrm{mVs}^{-1}$ and (b) areal capacitance at different scan rates of BGr symmetric and asymmetric SCs.

$\mathrm{mHz}$ to $1 \mathrm{MHz}$ with a potential amplitude of $10 \mathrm{mV}$ were carried out using an electrochemical workstation (Metrohm Autolab, PGSTAT302N), with two electrodes setup. The galvanostatic charging discharging (GCD) analysis at different current densities was studied by using a source meter (Agilent, U2722A) controlled with LabVIEW program. The capacitance, areal capacitance $\left(\mathrm{C}_{\mathrm{A}}\right)$, areal energy density $\left(\mathrm{E}_{\mathrm{A}}\right)$ and power density $\left(\mathrm{P}_{\mathrm{A}}\right)$ were measured from $\mathrm{CV}$ and $\mathrm{GCD}$ analysis using the equations reported in previous work [11]. The electrode with an area of $4 \mathrm{~cm}^{2}$ were used the fabrication of ASC.

\section{RESULTS AND DISCUSSIONS}

\section{A. Microstructural analysis}

The microstructure of the electrode materials has prominent role in determining the capacitance performance of the SCs [31-33]. In Figs. 1(c) to 1(e) show the microstructures of electrode/fabric materials Berlin fabric, graphite film and Nora Dell fabric respectively. The Berlin fabric shows the typical textile morphology, and the highly porous microstructure of printed graphite film is evident from Fig. 1(d). Such porous microstructure is highly desirable for $\mathrm{SC}$ electrode as it can increase the surface area and thereby the edlc $[34,35]$. In the case of Nora Dell fabric (Fig. 1(e)), the microstructure (after redox reaction) shows nano platelets like morphology of the oxides. During the electrochemical reaction, the $\mathrm{Ni}$ and $\mathrm{Cu}$ metals on the fabric undergo a redox reaction with electrolyte and form their respective oxides [1]. These oxides are responsible for the pseudocapacitance in the fabricated ASC.

\section{B. Electrochemical analysis of ASC}

During the charging of the fabricated ASC, the positive ions from the electrolyte (PVA-KCl) move towards graphite electrodes and form an electric double layer (edl). Likewise, in pseudocapacitor is formed towards the positive electrode as the interaction of negative ions from the electrolyte lead to the oxidation of copper and nickel while undergoing reversible redox reaction [1]. The electrochemical performances of these reaction in the developed ASC were evaluated using EIS and $\mathrm{CV}$ analysis. Fig. 2 shows the comparison of $\mathrm{CV}$ curve and areal capacitance of the BGr-ND ASC with BGr symmetric SCs (BGr-SySC). The CV curve of BGr-SySC shows (Fig. 2(a)) a rectangular shape with a small redox peak at $0.1 \mathrm{~V}$ (redox reaction of $\mathrm{Ag}$ ). At the same time, the BGr-ND ASC
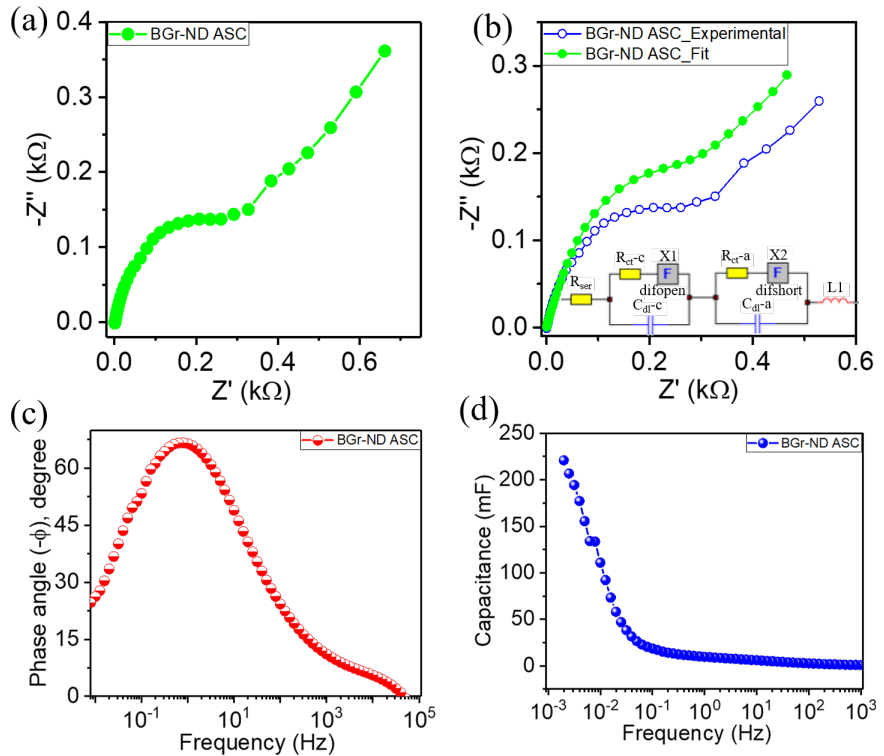

(d)

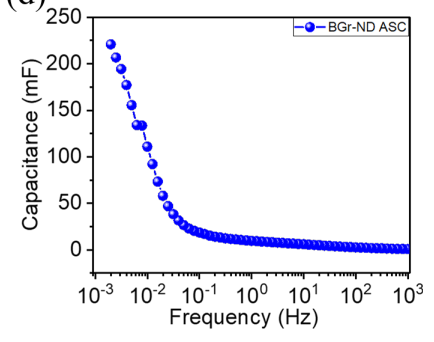

Fig. 3. (a) Nyquist plot (b) experimental and theoretically fitted Nyquist plot (fitting circuit inset) (c) Bode plot and (d) capacitance variation with frequency of BGr-ND ASC respectively.

shows a distorted rectangular shape because of combined edlc and pseudocapacitance. Also, it is evident that the area under the $\mathrm{CV}$ curve, which is a measure of energy storage and peak current, is high for BGr-ND ASC compared to symmetric SC. These results suggest that the combined effect of edlc and pseudocapacitance is responsible for improved capacitance of the ASC. This is also evident from the variation in areal capacitance with scan rate as represented in Fig. 2(b). For all scan rates the BGr-ND ASC shows higher areal capacitance than the BGr-SySC.

Fig. 3. Shows the results from the EIS analysis of BGr-ND ASC. The Nyquist plot of the ASC is given in Fig. 3(a). The high frequency region of the Nyquist plot gives the information about the contact resistance of the electrode material. The equivalent series resistance (ESR) of the ASC measured from here shows a very low value of $0.95 \Omega$ (at 1 $\mathrm{kHz}$ ). The low ESR indicates better conductivity of the electrode. The semicircle in the middle frequency region of the Nyquist plot represents the charge transfer resistance $\left(\mathrm{R}_{\mathrm{ct}}\right)$ and the sharp line at the lower frequency region indicates the diffusion and double layer capacitance formation $[1,2]$. To investigate the capacitance formation and contribution of each components to the total energy storage of the ASC, the electrochemical circuit fitting of the Nyquist plot was carried out using multiple EIS parameterization software [36]. The fitted curve and the circuit used are given in Fig. 3(b). Both curves show similar nature, and the fitted parameters are given in Table 1, especially resistive and capacitive components. The ESR $\left(R_{\text {ser }}\right)$ in Table 1 shows a value of $0.94 \Omega$, which is comparable with experimental results. The circuit used for

\begin{tabular}{|c|c|c|c|c|}
\hline & $\mathbf{R}_{\text {ser }}$ & $\mathbf{R}_{\mathbf{d}-\mathbf{c}}$ & $\mathbf{R}_{\mathrm{ct}-\mathbf{a}}$ & $\mathbf{R}_{\mathbf{d}-\mathbf{a}}$ \\
\hline \multirow[t]{2}{*}{ Resistive ( $(\Omega)$} & 0.94 & 1021.08 & 87.08 & 164.96 \\
\hline & $\mathbf{C}_{\mathrm{d}-\mathrm{c}}$ & $\mathrm{C}_{\mathrm{dl}-\mathrm{c}}$ & $\mathbf{C}_{\mathrm{d}-\mathrm{a}}$ & $\mathrm{C}_{\mathrm{dl}-\mathrm{a}}$ \\
\hline Capacitive (F) & 0.497 & 0.0282 & 0.0823 & .0038 \\
\hline
\end{tabular}



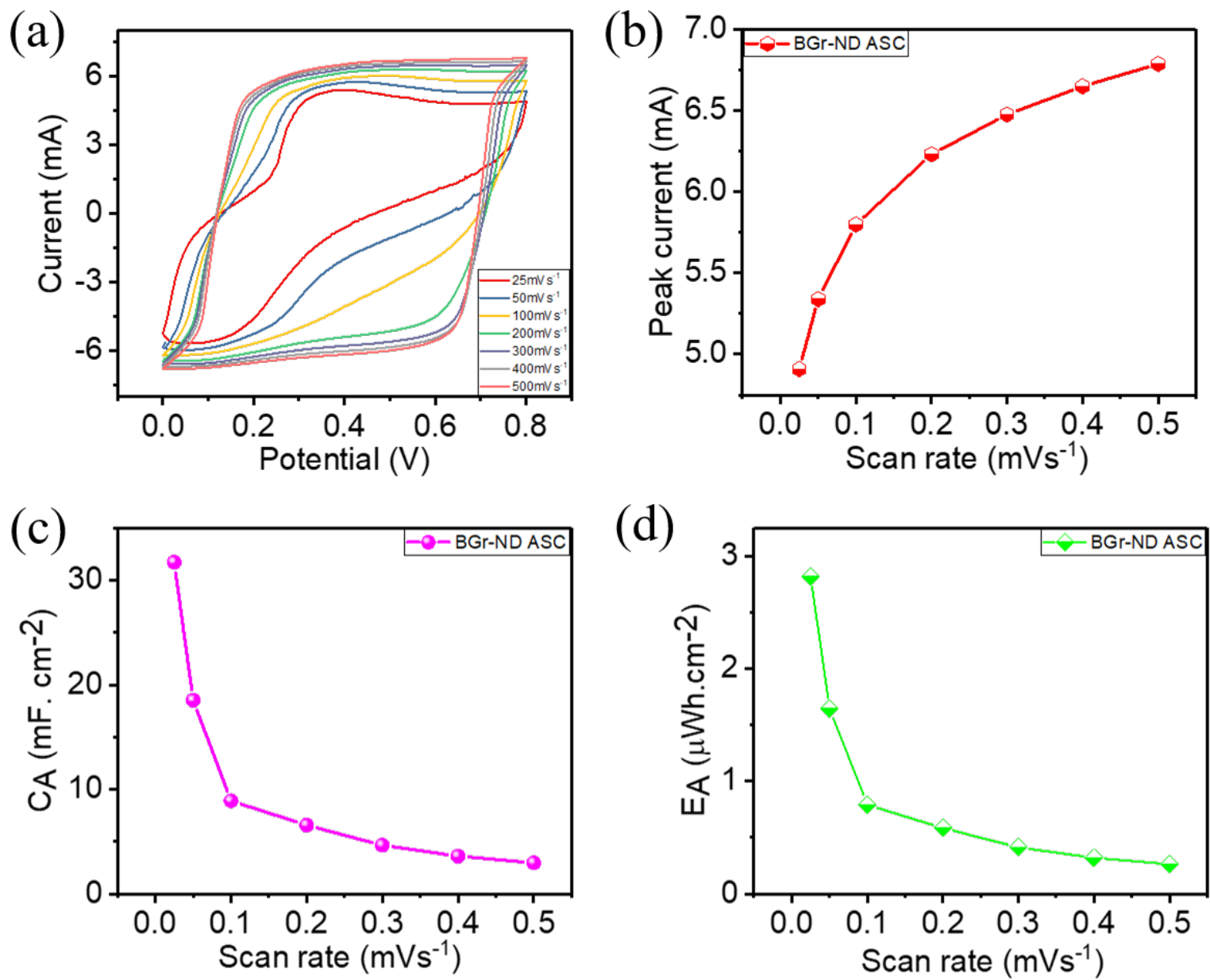

(d)

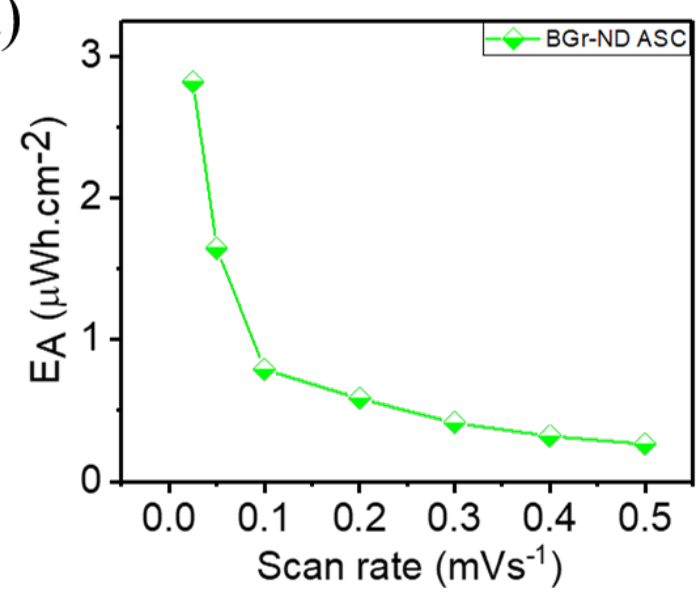

Fig. 4. (a) CV curves (b) peak current (c) areal capacitance and (d) areal energy density of BGr-ND ASC at different scan rate, respectively.

fitting is battery type with anodic and cathodic contributions. Comparing the double layer capacitance of both cathode and anode $\left(\mathrm{C}_{\mathrm{dl} \_\mathrm{c}}\right.$ and $\left.\mathrm{C}_{\mathrm{dl} \_} \mathrm{a}\right)$, the former shows an order of magnitude better capacitance $(28.2 \mathrm{mF})$ compared to anodic double layer capacitance $\left(\mathrm{C}_{\mathrm{dl} \_}, 3.8 \mathrm{mF}\right)$. This is expected because the primary capacitance formation in cathode (negative electrode) is edlc formation on the graphite layer. In the fitting, L1 represents the inductivity from either the winding or maybe due to an artifact which normally occurs in this type battery configuration. However, the value of the inductance is very low (1.01208e-014 H).

The Bode plot of the BGr-ND ASC is given in Fig. 3(c). The result shows a maximum phase angle of around $70^{\circ}$ in the lower frequency region indicating the capacitive behaviour of the device. The frequency dependent capacitance variation of BGr-ND ASC is shown in Fig. 3(d). The SC shows a capacitance value of around $225 \mathrm{mF}$ at a lower frequency of 2 $\mathrm{mHz}$ and it decreases with the frequency. The high capacitance at lower frequency is due to the intercalation of the ions in the porous graphite electrode as well as the redox reaction taking place in the Nora Dell fabric $(\mathrm{Cu} / \mathrm{Ni}$ metal oxides).

The CV analysis of the fabricated BGr-ND ASC is carried out up to $0.8 \mathrm{~V}$ under different scan rates varying from 25-500 $\mathrm{mVs}^{-1}$ and the respective plots are given in Fig.4(a). At the lower scan rate $\left(25 \mathrm{mVs}^{-1}\right)$, a sharp redox peak is evident in the $\mathrm{CV}$ curve at about $0.3 \mathrm{~V}$. As per our previous observations, the origin of this peak is lies in the redox reaction taking place in the $\mathrm{Cu} / \mathrm{Ni}$ metal in Nora Dell fabric due to the slower diffusion of ions from the electrolyte to the pores of the electrode material $[1,2]$. However, at higher scan rates the CV curves show rectangular shapes like that of an ideal double layer capacitor. This is due to the fast-ionic diffusion taking place at higher scan rate [2]. The peak current of the CV curves shows an increasing trend with scanning rates as shown in Fig. 4(b). Similarly, the variation in areal capacitance and energy density of ASC with different scan rates is given in Fig. 4(c) and 4(d) respectively. The device shows a maximum areal capacitance and energy density of $32 \mathrm{mF} . \mathrm{cm}^{-2}$ and 2.8 $\mu \mathrm{Wh} . \mathrm{cm}^{-2}$ respectively at a scan rate of $25 \mathrm{mV} . \mathrm{s}^{-1}$. Furthermore, with increasing scan rate both areal capacitance and energy density shows a decreasing trend. This obvious because at lower san rate the ions will go inside pores of the electrode and promote the redox reactions. However, due to the fast charging discharging cycles at higher scans the ions can only interact the electrode surface and lead to reduced double layer formation and redox reactions, eventually resulting in lower areal capacitance and energy density [1].

The GCD analysis of the BGr-ND ASC was performed up to $0.8 \mathrm{~V}$ with different applied currents ranging from $2-10 \mathrm{~mA}$ 

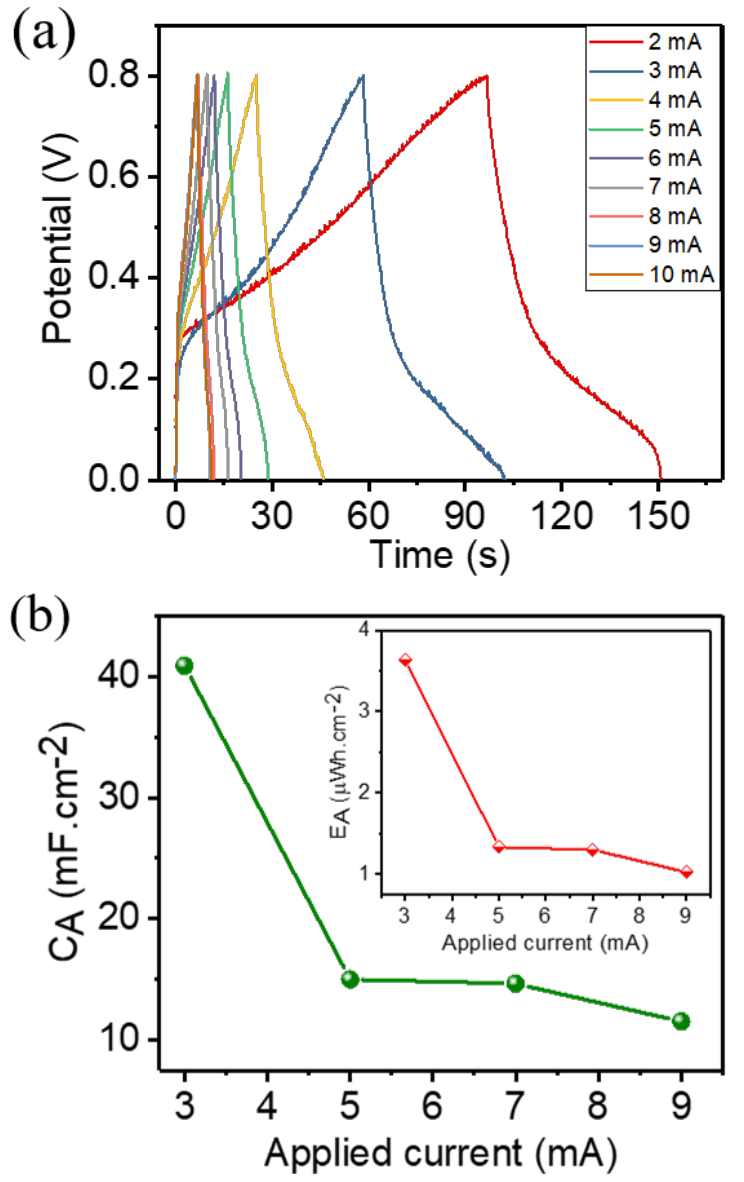

Fig. 5. (a) GCD curves at different applied current (b) areal capacitance and areal energy density (inset) of BGr-ND ASC respectively.

as shown in Fig. 5. Both the pseudocapacitive and the edlc nature of the GCD curve are evident from Fig. 5(a). Here one electrode exhibits more like pseudocapacitive/ battery type characteristics during charging discharging, but as whole the device shows capacitive nature with a large deviation from ideal capacitor [22]. The SC shows a low IR drift of 30-80 mV with this applied current. The areal capacitance and energy density of the ASC was calculated from the GCD curves and the results are shown in Fig. 5(b). The device has a high areal capacitance of $41 \mathrm{mF} . \mathrm{cm}^{-2}$ at an applied current density of $0.75 \mathrm{mAcm}^{-2}$. Interestingly, the device shows a capacitance of around $13 \mathrm{mF} \cdot \mathrm{cm}^{-2}$ at a high applied current of $10 \mathrm{~mA}$ indicating the high capacitive performance. We also measured the energy and power density of the ASC from GCD analysis. The inset in Fig. 5(b) shows the variation of energy density for different applied currents. The SC shows a high energy density of $3.6 \mu \mathrm{Wh} . \mathrm{cm}^{-2}$ at an applied current density of 0.75 $\mathrm{mA} . \mathrm{cm}^{-2}$. The power density of the ASC is also calculated at this applied current and it was $300 \mu \mathrm{W} . \mathrm{cm}^{-2}$.

The cyclic stability is an important consideration for practical applications and hence the fabricated device was evaluated for 5000 cycles with a high applied current of 10 $\mathrm{mA}$ and the results are depicted in Fig. 6. Fig. 6(a) shows the GCD curves of 5000 cycles and Fig. 6(b) and (c) show the first and last 10 cycles of the 5000 cycles, respectively. For the first 1000 cycles the device showed about $45 \%$ retention of capacitance and around 30\% retention for the rest of 5000 cycles. The comparatively low cyclic stability of the device may be due to the non-reversible reaction taking place in $\mathrm{Cu}$ and Ni metal in the Nora Dell fabric and due to electrode rupture occurred due to the insertion of large electrolyte ions $[1,37]$.

Wearable systems have recently attracted significant attention due to numerous applications which range from health monitoring to fashion and defense. From operational point of view, a critical component for effective use of wearable systems is the on-board energy devices to power various components. Recent studies demonstrate that skin or tattoo like sensory patches for health monitoring or robotic applications required an operating power in the range of nanowatt to microwatt $(\mathrm{nW}-\mu \mathrm{W})$ [38]. For example, the electrocardiogram (ECG) sensor which can operates in $\mathrm{nW}$
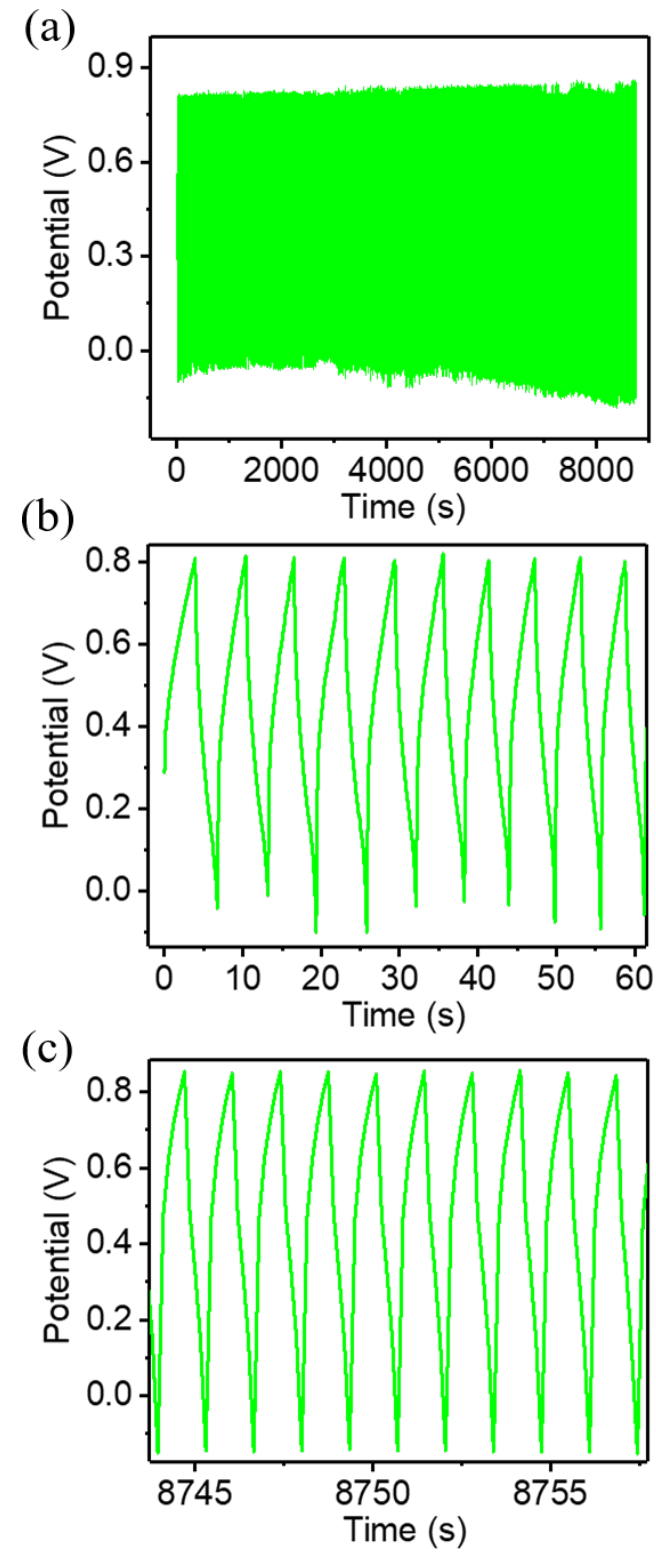

Fig. 6. (a) 5000 charging-discharging cycles (b) and (c) first and last 10 cycles of charging-discharging of BGr-ND ASC, respectively. 


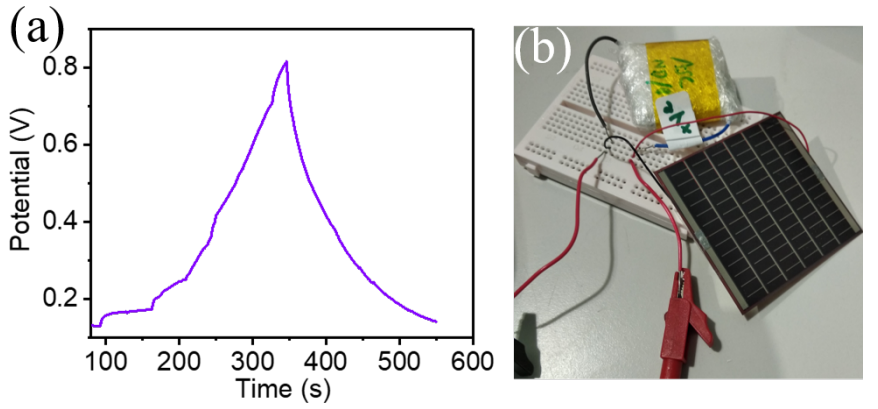

Fig. 7. (a) Charging of BGr-ND ASC with flexible solar cell and its selfdischarging (b) photographs of charging setup.

power and similarly the low power temperature sensor that operates at $\mathrm{pW}$ can be powered using the presented ASC [39, 40]. We observed that the fabricated ASC has an energy density of $3.6 \mu \mathrm{Wh} . \mathrm{cm}^{-2}$ and operating potential of about $0.8 \mathrm{~V}$. Further, by integrating multiple devices (series or parallel) we can tune the energy density via increasing the capacitance or operating potential window. Considering this, the ASC in textiles is excellent device for adoption in the wearable systems. One of the major requirements in this type of ASCs is viable method for charging the SC. For wearable and self-charging application as a proof of concept we demonstrated the charging of developed BGr-ND ASC with a flexible solar cell as shown in Fig. 7. Fig. 7(a) shows the charging of BGr-ND ASC with flexible solar cell (amorphous Si based solar cell) up to $0.8 \mathrm{~V}$ and its self-discharging. The photographs of the charging set up is given in Fig. 7(b). This proof-of-concept arrangement shows that by integrating the presented ASC with cloths, gloves and woven fabrics, it is possible to power the sensors and other components on wearable systems.

\section{CONCLUSION}

A metal coated fabric-based ASC is presented in this paper as a solution to power various devices on the wearable systems. The ASC consist of graphite paste printed on Berlin fabric as the negative electrode and $\mathrm{Cu} / \mathrm{Ni} / \mathrm{Ag}$ plated conductive fabric called Nora Dell as the positive electrode. The presented ASC uses the biocompatible PVA-KCl gel electrolyte as the safe and sustainable alternative. The microstructural analysis of the electrode materials shows highly porous structure with nano size metal oxides particles that contribute to the high capacitive behaviour of the fabricated device. The electrochemical studies and comparison of the obtained areal capacitance and energy density (41 $\mathrm{mF} . \mathrm{cm}^{-2}$ and $3.6 \mu \mathrm{Wh} . \mathrm{cm}^{-2}$ respectively at an applied current density of $0.75 \mathrm{~mA} . \mathrm{cm}^{-2}$ ) with symmetric supercapcitors shows better performance for the BGr-ND ASC. The longterm cyclic stability and self-charging with flexible solar cells confirm the suitability of the fabricated ASC for wearable applications.

\section{ACKNOWLEDGMENT}

This work was supported in part by Engineering and
Physical Sciences Research Council (EPSRC) Fellowship for Growth (EP/M002527/1 and EP/R029644/1), the Royal Society and the Science and Engineering Research Board of India (SERB) through Newton International Fellowship (NIF\R1\182437), and European Commission through Aquasense project (H2020-MSCA-ITN-2018-813680).

\section{REFERENCES}

M. S. Javed, N. Shaheen, S. Hussain et al., "An ultra-high energy density flexible asymmetric supercapacitor based on hierarchical fabric decorated with 2D bimetallic oxide nanosheets and MOFderived porous carbon polyhedra," J. Mater. Chem. A, vol. 7, no. 3, pp. 946-957, 2019.

A. Pullanchiyodan, L. Manjakkal, S. Dervin et al., "Metal Coated Conductive Fabrics with Graphite Electrodes and Biocompatible Gel Electrolyte for Wearable Supercapacitors," Adv. Mater. Technol., vol. 5, no. 5, pp. 1901107, 2020.

L. Manjakkal, A. Pullanchiyodan, N. Yogeswaran et al., "A Wearable Supercapacitor Based on Conductive PEDOT: PSS-Coated Cloth and a Sweat Electrolyte," Adv. Mater., pp. 1907254, 2020.

H. Peng, G. Qian, N. Li et al., "Flexible Asymmetric Supercapacitors with Ultrahigh Energy Density through Synergistic Design of Electrodes," $A d v$. Sci., vol. 5, no. 11, pp. 1800784, Nov, 2018.

R. Dahiya, N. Yogeswaran, F. Liu et al., "Large-Area Soft e-Skin: The Challenges Beyond Sensor Designs," Proc. IEEE, vol. 107, no. 10, pp. 2016-2033, 2019.

Manjakkal, S. Dervin, and R. Dahiya, "Flexible potentiometric pH sensors for wearable systems," $R S C A d v$., vol. 10, no. 15, pp. 8594-8617, 2020.

N. Wang, X. Wang, $Y$. Zhang asymmetric supercapacitor based on composite of polypyrrolegraphene oxide and poly(3,4-ethylenedioxythiophene)," J. Alloys Compd., vol. 835, 2020.

J. Zhao, C. Li, Q. Zhang et al., "An all-solid-state, lightweight, and flexible asymmetric supercapacitor based on cabbage-like $\mathrm{ZnCo}_{2} \mathrm{O}_{4}$ and porous $\mathrm{VN}$ nanowires electrode materials," J. Mater. Chem. A, vol. 5, no. 15, pp. 6928-6936, 2017.

T. An, and W. Cheng, "Recent progress in stretchable supercapacitors," J. Mater. Chem. A, vol. 6, no. 32, pp. 1547815494, 2018.

C. Choi, J. A. Lee, A. Y. Choi et al., "Flexible supercapacitor made of carbon nanotube yarn with internal pores," Adv. Mater., vol. 26, no. 13, pp. 2059-65, 2014.

S. Zhang, and N. Pan, "Supercapacitors Performance Evaluation," Adv. Energy Mater., vol. 5, no. 6, 2015.

L. Manjakkal, C. G. Núñez, W. Dang et al., "Flexible self-charging supercapacitor based on graphene-Ag-3D graphene foam electrodes," Nano Energy, vol. 51, pp. 604-612, 2018.

L. Manjakkal, W. T. Navaraj, C. G. Nunez et al., "GrapheneGraphite Polyurethane Composite Based High-Energy Density Flexible Supercapacitors," Adv. Sci., vol. 6, no. 7, pp. 1802251, 2019.

K. Jost, G. Dion, and Y. Gogotsi, "Textile energy storage in perspective," J. Mater. Chem. A, vol. 2, no. 28, pp. 10776-10787, 2014.

S. Zhai, H. E. Karahan, L. Wei et al., "Textile energy storage: Structural design concepts, material selection and future perspectives," Energy Stor. Mater., vol. 3, pp. 123-139, 2016.

M. Liu, Z. Cong, X. Pu et al., "High-Energy Asymmetric Funct. Mater., vol. 29, no. 41, 2019.

K. Dong, Y. C. Wang, J. Deng et al., "A Highly Stretchable and Washable All-Yarn-Based Self-Charging Knitting Power Textile Composed of Fiber Triboelectric Nanogenerators and Supercapacitors," ACS Nano, vol. 11, no. 9, pp. 9490-9499, 2017. L. Manjakkal, W. Dang, N. Yogeswaran et al., "Textile-Based Potentiometric Electrochemical $\mathrm{pH}$ Sensor for Wearable Applications," Biosensors, vol. 9, no. 1, pp. 14, 2019.$$
\text { Pp. 946-957, } 2019 .
$$ 
[19] S. Jiao, T. Li, C. Xiong et al., "A Facile Method of Preparing the Asymmetric Supercapacitor with Two Electrodes Assembled on a Sheet of Filter Paper," Nanomaterials (Basel), vol. 9, no. 9, 2019.

[20] S. Jin, H. Lee, and S. Yim, "Enhanced capacitive properties of allmetal-oxide-nanoparticle-based asymmetric supercapacitors," $R S C$ $A d v$., vol. 9, no. 55, pp. 31846-31852, 2019.

[21] W. Liu, M. Zhu, J. Liu et al., "Flexible asymmetric supercapacitor with high energy density based on optimized $\mathrm{MnO}_{2}$ cathode and $\mathrm{Fe}_{2} \mathrm{O}_{3}$ anode," Chin. Chem. Lett., vol. 30, no. 3, pp. 750-756, 2019.

[22] Y. Shao, M. F. El-Kady, J. Sun et al., "Design and Mechanisms of Asymmetric Supercapacitors," Chem. Rev., vol. 118, no. 18, pp. 9233-9280, 2018.

[23] H. Han, S. W. Lee, K. H. Moon et al., "Fabrication of Solid-State Asymmetric Supercapacitors Based on Aniline Oligomers and Graphene Electrodes with Enhanced Electrochemical Performances," ACS Omega, vol. 4, no. 1, pp. 1244-1253, 2019.

[24] H. Gao, F. Xiao, C. B. Ching et al., "High-performance asymmetric supercapacitor based on graphene hydrogel and nanostructured $\mathrm{MnO}_{2}$," ACS Appl. Mater. Interfaces, vol. 4, no. 5, pp. 2801-10, 2012.

[25] M. A. Garakani, S. Abouali, Z.-L. Xu et al., "Heterogeneous, mesoporous $\mathrm{NiCo}_{2} \mathrm{O}_{4}-\mathrm{MnO}_{2} /$ graphene foam for asymmetric supercapacitors with ultrahigh specific energies," J. Mater. Chem. $A$, vol. 5, no. 7, pp. 3547-3557, 2017.

[26] P. Sundriyal, and S. Bhattacharya, "Textile-based supercapacitors for flexible and wearable electronic applications," Sci. Rep., vol. 10 , no. 1 , pp. $13259,2020$.

[27] S. Lei, Y. Liu, L. Fei et al., "Commercial Dacron cloth supported $\mathrm{Cu}(\mathrm{OH})_{2}$ nanobelt arrays for wearable supercapacitors," J. Mater. Chem. A, vol. 4, no. 38, pp. 14781-14788, 2016.

[28] A. Pullanchiyodan, L. Manjakkal, and R. Dahiya, "Metal Coated Fabric Based Supercapacitors." in Proc. IEEE Int. Conf. Flexible Printable Sensors Syst. (FLEPS), Aug. 2020, pp. 1-4.

[29] M. Ntagios, H. Nassar, A. Pullanchiyodan et al., "Robotic hands with intrinsic tactile sensing via 3D printed soft pressure sensors," Adv. Intell. Syst., pp. 1900080, 2019.

[30] L. Manjakkal, D. Shakthivel, and R. Dahiya, "Flexible Printed Reference Electrodes for Electrochemical Applications," $A d v$. Mater. Technol., vol. 3, no. 12, pp. 1800252, 2018.

[31] O. Ghodbane, J.-L. Pascal, and F. Favier, "Microstructural Effects on Charge-Storage Properties in $\mathrm{MnO}_{2}$-Based Electrochemical Supercapacitors," ACS Appl. Mater. Interfaces, vol. 1, no. 5, pp. 1130-1139, 2009.

[32] D. Wang, Z. Lu, and L. Xu, "Microstructure design of porous nanocarbons for ultrahigh-energy and power density supercapacitors in ionic liquid electrolyte," J. Mater. Sci., vol. 55, no. 17, pp. 7477-7491, 2020.

[33] R. Kumar, P. Rai, and A. Sharma, "Facile synthesis of $\mathrm{Cu}_{2} \mathrm{O}$ microstructures and their morphology dependent electrochemical supercapacitor properties," RSC Adv., vol. 6, no. 5, pp. 3815-3822, 2016.

[34] R. Heimböckel, F. Hoffmann, and M. Fröba, "Insights into the influence of the pore size and surface area of activated carbons on the energy storage of electric double layer capacitors with a new potentially universally applicable capacitor model," Phys. Chem. Chem. Phys., vol. 21, no. 6, pp. 3122-3133, 2019.

[35] R. Härmas, R. Palm, M. Härmas et al., "Influence of porosity parameters and electrolyte chemical composition on the power densities of non-aqueous and ionic liquid based supercapacitors," Electrochim. Acta, vol. 283, pp. 931-948, 2018.

[36] L. Manjakkal, E. Djurdjic, K. Cvejin et al., "Electrochemical Impedance Spectroscopic Analysis of $\mathrm{RuO}_{2}$ Based Thick Film $\mathrm{pH}$ Sensors," Electrochim. Acta, vol. 168, pp. 246-255, 2015.

[37] J. Zhu, Y. Xu, J. Wang et al., "The effect of various electrolyte cations on electrochemical performance of polypyrrole/RGO based supercapacitors," Phys. Chem. Chem. Phys., vol. 17, no. 43, pp. 28666-73, 2015.

[38] C. García Núñez, L. Manjakkal, and R. Dahiya, "Energy autonomous electronic skin," npj Flex. Electron., vol. 3, no. 1, pp. $1,2019$.

[39] H. Wang, and P. P. Mercier, "Near-Zero-Power Temperature Sensing via Tunneling Currents Through Complementary MetalOxide-Semiconductor Transistors," Sci. Rep., vol. 7, no. 1, pp. 4427, 2017.
[40]

T. Tekeste, H. Saleh, B. Mohammad et al., "A Nanowatt RealTime Cardiac Autonomic Neuropathy Detector," IEEE Trans Biomed. Circuits Syst. vol. 12, no. 4, pp. 739-750, 2018.

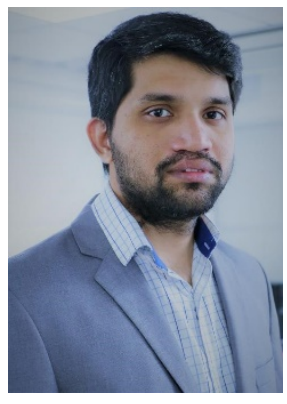

Abhilash Pullanchiyodan received B.Sc. and M.Sc. degree in Chemistry, from Kannur University, Kerala, India, and Ph.D. degree in Chemical Science (Chemistry) in 2018, from Academy of Scientific and Innovative Research (AcSIR), at National Institute for Interdisciplinary Science and Technology, (CSIR-NIIST), Trivandrum, India. From 20182019 he worked as postdoctoral research assistant, and since 2019 is working as a Royal Society-SERB Newton International Fellow in BEST group at University of Glasgow (UK). He authored/co-authored 20 journal papers and 5 conference proceedings. His research interest includes supercapacitors, nanomaterial synthesis, ink/paste formulation, microwave dielectrics, ceramics, and nanocomposite preparation.

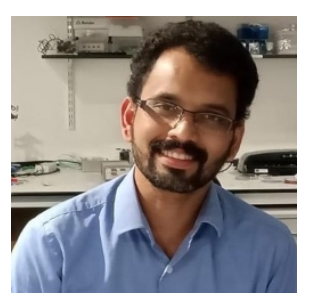

Libu Manjakkal received B.Sc. and M.Sc. degree in physics from Calicut University, and Mahatma Gandhi University, India and Ph.D. degree in Electronic Engineering from Institute of Electron Technology (ITE), Poland, in 2012-2015 (Marie Curie ITN Program). From 2009 to 2012 he was worked at CMET, India. In 2012, he was with New University of Lisbon, Portugal. From 2015 to 2016, he was a post-doctoral with ITE. Since 2016, he was a postdoctoral at BEST group in the University of Glasgow, UK. He has authored/co-authored more than 60 scientific papers. His research interest includes material synthesis, electrochemical technology, sensors, flexible electronics, supercapacitors and energy autonomous systems.

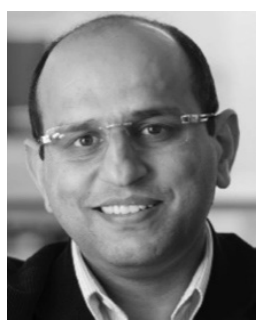

Ravinder Dahiya is Professor of Electronics and Nanoengineering in the University of Glasgow, U.K. He is the leader of Bendable Electronics and Sensing Technologies (BEST) research group. His group conducts fundamental and applied research in flexible and printable electronics, tactile sensing, electronic skin, robotics, and wearable systems. $\mathrm{He}$ has authored over 350 research articles, 7 books, and 15 submitted/granted patents. He has led several international projects. He is President-Elect (2020-21) and Distinguished Lecturer of the IEEE Sensors Council and is serving on the editorial boards of the Scientific Report. He was also on the editorial boards of IEEE SENSORS JOURNAL (2012-2020) and IEEE TRANSACTIONS ON ROBOTICS (2012-2017). He was the Technical Program co-chair of IEEE Sensors 2017 and IEEE Sensors 2018 and has been General Chair of several conferences including IEEE FLEPS (2019, 2020, 2021), which he founded in 2019. He holds the prestigious EPSRC Fellowship and received in past the Marie Curie and Japanese Monbusho Fellowships. He has received several awards, including 2016 Microelectronic Engineering Young Investigator Award (Elsevier), 2016 Technical Achievement Award from the IEEE Sensors Council and 9 best paper awards as author/coauthor in International Conferences and Journals. 